\title{
Value of pretreatment prognostic nutritional index in predicting intravenous immunoglobin-resistant Kawasaki disease
}

\author{
Gang $\mathrm{Li}^{1}$, Xiumei $\mathrm{Xu}^{1}$, Pengyuan $\mathrm{Chen}^{2}$, Yongying Gou ${ }^{1}$, Rumeng Zeng ${ }^{1}$, Dong $\mathrm{Liu}^{1}$, Yan \\ Duan $^{1}$, Ting Wang ${ }^{1}$, Peng Jia ${ }^{1}$, and Bin Liu ${ }^{1}$ \\ ${ }^{1}$ The Affiliated Hospital of Southwest Medical University \\ ${ }^{2}$ Sichuan Academy of Medical Sciences and Sichuan People's Hospital
}

May 29, 2020

\begin{abstract}
Background: The aim of the present study was to investigate the potential predictive significance of pretreatment prognostic nutritional index (PNI) in patients with intravenous immunoglobulin (IVIG) resistant Kawasaki disease (KD). Methods: From June 2013 to May 2020, 1,257 eligible patients with KD were included in the present study. The pretreatment PNI was calculated as albumin level $(\mathrm{g} / \mathrm{L})+5 \times$ total lymphocyte count $(109 / \mathrm{L})$. The optimal cut-off values for PNI, neutrophil to lymphocyte ratio (NLR), and platelet to lymphocyte ratio (PLR) were evaluated via a receiver operating curve analysis. The impact of pretreatment PNI, NLR and PLR for IVIG resistant KD were tested with the Student's t test or Mann-Whitney U test, and univariate and multivariate analyses. Results: The optimal cut-off values were identified as 49.50 for PNI, 3.58 for NLR and 164.00 for PLR, respectively. Lower pretreatment PNI levels were demonstrated to be associated with lower age, serum sodium levels and platelet counts, and with a higher incidence of IVIG resistance and higher C-reactive protein levels. There was a significantly negative association between the PNI and NLR, and PLR. In the logistic analyses, PNI as independent predictive factors were significantly correlated with IVIG resistance. The discriminatory ability of PNI was not inferior to NLR and PLR for predicting IVIG resistance. Conclusion: Pretreatment PNI could serve as a novel surrogate independent predictor for patients with IVIG resistant KD.
\end{abstract}

\section{Background}

Kawasaki disease (KD) is an acute febrile illness characterized by multisystemic vasculitis, which usually may be associated with coronary artery lesions (CALs), and has been considered as the most common cause of acquired heart disease among children in industrialized countries. Although the treatment of intravenous immunoglobulin (IVIG) plus aspirin has greatly decreased the incidence of CALs within the first 10 days of fever onset, previous studies indicated that 10-20\% of patients with KD were unresponsive to initial treatment with IVIG, and even 20-30\% of patients resistant to initial treatment with IVIG did not respond to a second dose of IVIG [1, 2]. This condition could be more prone to risk of CALs.

Increasing evidence has confirmed that systemic inflammation and immune responses play a crucial role in the pathogenesis and progression of KD. Several prognostic models for IVIG resistant KD have been widely investigated, such as the Formosa scoring system from Taiwan, China, the San Diego scoring system from American, and the Kobayashi, Egami and Sano scoring system from Japan. However, these models have been relatively lacking in the Mainland of China [3]. A large number of studies also attempted to search markers for predicting IVIG resistant KD. Recently, it was reported that the peripheral biomarker of immunity/inflammation, the neutrophil-to-lymphocyte ratio (NLR), and platelet (PLT)-to-lymphocyte ratio (PLR) were identified as significant prognostic indicators in KD with IVIG resistance [4-6]. In addition, serum albumin, known as an indicator of host inflammatory and nutritional status, had been confirmed to have a 
prognostic role in the Chinese population with IVIG resistant KD [7]. However, these models or indicators were not satisfactory for predicting IVIG resistance in clinical practice. Recently, it has been hypothesized that immunonutritional status could reflect the general condition of patients, including immunocompetence, protein turnover and physical condition. The prognostic nutritional index (PNI) based on a combination of albumin levels and peripheral blood lymphocyte count, was supposed to show a more accurate representation of the nutritional and immunological status in various types of cancer [8]. Its prognostic value in various types of tumor, such as urinary cancer, tongue squamous cell carcinoma [9], osteosarcoma [10], gynecological cancer [11], non-small cell lung cancer[12] and pulmonary embolism[13], acute myocardial infarction[14, 15], idiopathic dilated cardiomyopathy [16] and heart failure [17] has been widely investigated. Recently, Tai et al reported that a low pre-treatment PNI level $(\mathrm{PNI}<55)$ correlated to a high incidence of CALs, as well as IVIG non-responder in 275 children with KD from Taiwan [18], but the sample size was small and cases were came from only one institution. Therefore, the present study was designed in order to further determine whether PNI could predict IVIG resistance in large cohort of patients with KD from six institutions.

\section{Methods}

\section{Study population}

A total of 1,257 patients with KD at six institutions between June 2013 and May 2020 were retrospectively analyzed in the present study. The diagnostic criteria for KD conformed to the guidelines of the AHA 2004[19], and subsequently, by the AHA 2017[20]. The exclusion criteria were as follows: (1) History of KD; (2) incident cases in receipt of IVIG treatment in another hospital; (3) non-use of IVIG during hospitalization; (4) poor laboratory data records during hospitalization; and (5) the patients with inflammatory diseases, immune diseases, hematological diseases, metabolic diseases, cancer, liver and kidney diseases, and other heart diseases. All children with KD were treated with IVIG $[1-2 \mathrm{~g} /(\mathrm{kg} /$ day $)]$ plus oral aspirin $[30-50 \mathrm{mg} /(\mathrm{kg} /$ day $)]$. IVIG-resistant patients were those with recurrent fever [?]38 ${ }^{\circ} \mathrm{C}$ and recrudescence of one or more of the initial symptoms, or persistent fever for at least $36 \mathrm{~h}$ after completion of initial IVIG infusion [19]. The present study was approved by the Ethics Committee of the Affiliated Hospital of Southwest Medical University, Sichuan Academy of Medical Sciences, Sichuan People's Hospital, The First People's Hospital of Yibin, The Second People's Hospital of Yibin, Luzhou People's Hospital, Southwest Medical University Affiliated Hospital of traditional Chinese Medicine, which strictly complied with the 1975 Helsinki Declaration, revised in 1996.

The clinical parameters of patients, such as age and sex, were collected from the medical data. Laboratory investigations included the following: The counts of white blood cells, neutrophils, lymphocytes, monocytes and platelets, hemoglobin level, albumin level, serum sodium level and total bilirubin were collected before IVIG treatment. The definitions of PNI, NLR and PLR were shown as follows: PNI $=$ albumin $(\mathrm{g} / \mathrm{L})+$ $5 \times$ total lymphocyte count $\left(10^{9} / \mathrm{L}\right) ; \mathrm{NLR}=$ neutrophil/lymphocyte counts; and PLR = platelet/lymphocyte counts [10].

\section{Statistical analysis}

SPSS software (version 22.0) was used to perform the statistical analyses. Categorical variables were reported as frequencies and percentages, and were analyzed using $\chi^{2}$ tests. Continuous variables were analyzed using the Student's t test or Mann-Whitney U test, where appropriate. The receiver operating characteristic (ROC) curve analysis was utilized to determine the optimal cut-off values of inflammatory predictors with MedCalc 17.6. The area under the ROC curve (AUC) was used to compare prognostic factors. The multivariate logistic regression analysis was performed on the factors that were shown to be significant on univariate logistic analysis. Odds ratio (OR) and $95 \%$ confidence intervals (CIs) were utilized to evaluate relative risk. All tests were two-sided and $\mathrm{P}<0.05$ was considered to indicate a statistically significant different.

\section{Results}

\section{Patient characteristics}

The present study enrolled a total of 1,275 patients with KD with a median age of 19 months (range, 11-38 months) according to the inclusion criteria. Of these, 718 (57.1\%) were male and 539 (42.9\%) were female. 
There were $142(11.3 \%)$ patients with CALs and 187 (14.9\%) patients with IVIG resistance in all patients with KD. The clinical laboratory data are presented in Table 1.

\section{Optimal cut-off values of PNI, NLR and PLR for predicting IVIG resistance}

The AUC for IVIG resistance with a ROC analysis method were 0.707 (95\% CI, 0.681-0.732) $(p<0.001)$, 0.689 (95\% CI: $0.663-0.715)(p<0.001)$, and 0.676 (95\% CI: $0.650-0.702)(p<0.001)$ for PNI, NLR, and PLR, respectively. According to the maximum Youden index, the optimal cut-off values for predicting IVIG resistance were identified as 49.5 for PNI, 3.58 for NLR and 164 for PLR, . Pretreatment PNI was not inferior to NLR $(\mathrm{z}=0.868, p=0.385)$ and PLR $(\mathrm{z}=0.1764, p=0.078)$ for predicting IVIG resistance (Fig 1).

Accordingly, patients were divided into two groups according to the optimal cut-off values: PNI $<49.5$ $(\mathrm{n}=500)$ and PNI [?]49.5 $(\mathrm{n}=757)$ groups; NLR [?]3.58 $(\mathrm{n}=652)$ and NLR<3.58 $(\mathrm{n}=605)$ groups; PLR [?]164 $(\mathrm{n}=686)$ and PLR $<164(\mathrm{n}=571)$ groups.

\section{Association between clinical characteristics and PNI, NLR and PLR in KD}

The present study revealed the association between the inflammation-based biomarkers and clinical features of KD, which are all indicators of IVIG resistance (Table 2). The results revealed that pretreatment PNI was significantly associated with age $(p=0.0448), \operatorname{PLT}(p=0.0169)$ and serum sodium levels $(p=0.0163)$. The patients with low PNI tended to exhibit higher levels of C-reactive protein (CRP) $(p<0.0001)$ and a higher the incidence of IVIG resistance $(p=0.0042)$ when compared with the patients with high PNI. Furthermore, high NLR was significantly associated with low age $(p=0.0285)$, platelet $(p=0.0157)$ and serum sodium levels $(p=0.0086)$. A positive association between NLR and CRP $(p=0.0032)$, and the incidence of IVIG resistance $(p=0.0006)$ was detected in the present study. Nevertheless, no significant associations were observed between the two groups in terms of gender and total bilirubin level. In addition, high PLR has been demonstrated to be associated with high levels of CRP $(p=0.0001)$, higher incidence of IVIG resistance $(p=0.0116)$ and with low age $(p=0.0232)$. Nevertheless, no great differences between PLR and gender, total bilirubin, PLT and serum sodium levels were observed.

Notably, PNI was negatively correlated with NLR $(r=-0.4216 ; p<0.0001)$ and PLR $(r=-0.4669, p<0.0001)$ (Fig. 2). Thus, the results of the present study suggested that PNI was associated with other inflammatory biomarkers in $\mathrm{KD}$.

\section{Univariate and multivariate analyses for IVIG resistance}

In order to investigate the predictive value of pretreatment NLR, PLR and PNI, the present study performed univariate and multivariate analyses. In the univariate analysis, age $(p=0.031)$, platelet $(\mathrm{p}=0.004)$, CRP $(p<0.001)$, serum sodium levels $(p=0.028)$, NLR $(p=0.003)$, PLR $(p=0.009)$, and PNI $(p<0.001)$ were significantly associated with IVIG resistance. Furthermore, clinical features for IVIG resistance were also investigated in a multivariate analysis. The results suggested that age $(p=0.024)$, CRP $(p=0.006)$, serum sodium levels $(p=0.007)$, NLR $(p=0.008)$, PLR $(p=0.026)$, and PNI $(p=0.001)$ were still identified as markedly independent parameters for IVIG resistance (Table 3 ).

\section{Discussion}

To the best of our knowledge, the present study demonstrated for the first time using a large cohort of patients with KD in multicenter that pretreatment PNI was negatively associated with biomarkers of immunity/inflammation (NLR and PLR) and positively associated with age, platelet, serum sodium. Low PNI was significantly associated with higher levels of CRP and higher the incidence of IVIG resistance. As with NLR and PLR, PNI was an independent predictor of IVIG resistance. Pretreatment PNI was not inferior to NLR and PLR for predicting IVIG resistance.

In the present study, the percentage of IVIG-resistance was $14.9 \%$, which was far higher the $6.25 \%$ determined by Xu-Hai Tan et al[7] and was consistent with the 10-20\% reported by Fukunishiet al [21] and Sleeper et al [22]. The discrepancy could be attributed to the different study populations, the sample size and the 
definition of IVIG-resistance. IVIG-resistant KD has been observed to be associated with a higher incidence of CALs, which is detrimental to the outcome of KD. Early additional treatment before the initial use of IVIG could decrease the occurrence of CALs in IVIG resistant KD. Therefore, it is essential to investigate simple and feasible indictors for IVIG resistance.

Consistent with previous data [4-6], the markers of inflammatory and immunological activation, NLR and PLR, were associated with IVIG resistance in the present study. Systematic immunonutritional status has been refined by introduction of PNI, a continuous variable based on serum albumin concentration and total lymphocyte count in peripheral blood, which was associated with the mortality and morbidity in patients with cardiac diseases [23], acute kidney injury [24] and cancer [10]. The present study revealed that low PNI was significantly associated with high levels of CRP and low counts of platelet. Furthermore, an inverse correlation between PNI and the biomarkers of immunity/inflammation (NLR and PLR) was observed in the present study, which was in accordance with patients with osteosarcoma [10]. PNI was also demonstrated to be correlated with disease activity in patients with systemic lupus erythematosus [25, 26]. Besides, several previous studies reported that hyponatremia was observed in children with KD, particularly children with IVIG resistant KD [27-29] and was associated with the extent of inflammation [30, 31]. In line with these studies, low PNI was demonstrated to be closely associated with low serum sodium in the present study. These results clearly convey the notion that PNI may be associated with IVIG resistance.

PNI brings two important measurements together. On the one hand, serum albumin is known as an indicator of host inflammatory and nutritional status [8], and its levels are associated with higher mortality in several different types of tumor. Hypoalbuminemia was observed in IVIG resistant KD [32] and has been confirmed to be also closely associated with inflammatory markers, such as NLR and PLR. Additionally, research has shown that malnutrition was associated with the immune-suppressed condition [33, 34], impaired respiratory function, and poor wound healing [24], as well as increased risks of postoperative morbidity and mortality and prolonged hospital stays[24]. On the other hand, lymphocytopenia is regarded as the marker of a depressed cell-mediated immunity [35], which can lead to the development of systemic inflammatory response syndrome and is closely associated with immunity and inflammation. It has been reported that critically ill patients with shock and sepsis had marked lymphocytopenia, and that the severity of the clinical course was correlated with the divergence of the lymphocyte (lower) counts [35]. Lymphocytopenia was also associated with decreased survival in advanced carcinoma, sarcoma and lymphoma [36], similar to hypoalbuminemia. Numerous studies from large samples of multiple research centers in Asia have indicated that lymphocyte counts were significantly decreased in IVIG resistant KD when compared with IVIG responsive KD [7, $37,38]$. In the present study, low PNI was associated with decreased albumin and/or lymphocytes. The patients with lower PNI had significantly higher the incidence of IVIG resistance in the present study, which was consistent with previous findings [18]. According to these results, PNI, combined with albumin and lymphocyte levels, could be a superior index that reflects inflammation, immune system and nutritional status in IVIG resistant KD.

Valid prediction of the IVIG-treatment response is crucial to the patients with KD. A number of clinical and inflammatory indexes (age $<12$ months, platelet $<300 \times 10^{9} / \mathrm{L}$, higher total bilirubin levels and CRP levels, serum sodium levels, NLR and PLR) for predicting IVIG resistance have been reported [3, 7, 37, 39]. The results of the present study further confirmed those indictors (age $<12$ months, serum sodium, CRP, NLR and PLR), implying that the results of the present study are convincing. Regretfully, the aforementioned parameters had relatively low predictive power for IVIG resistance according to previous investigations. The prognostic value of PNI has been investigated in certain types of human cancer [9, 10], postoperative septic complications, ST segment elevation myocardial infarction [14]. PNI can also predict the clinical outcome of the pediatric population in the intensive care unit after cardiac operation [40].Accordingly, both univariate and multivariate analyses suggested that pretreatment low PNI was an independent predictor for IVIG resistance in the present study. Although the discriminatory ability of NLR and PLR to predict IVIG resistant $\mathrm{KD}$ has been demonstrated in previous reports $[5,6,37]$, as well as in the present study, pretreatment PNI having the highest AUC was not inferior to NLR and PLR as an indicator for predicting IVIG resistance determined by ROC analysis. The investigation performed by Miriliet al [41] in malignant 
melanoma was in accordance with the results of the present study. In addition, pretreatment NLR, PLR and PNI are emerging as predictors of the treatment response to neoadjuvant chemoradiotherapy in rectal cancer [42]. Taken together, PNI is a potential as a candidate for predicting IVIG resistance. As PNI is routinely available and can be measured accurately, it may become a useful and inexpensive approach to the IVIG-treatment response prediction in KD. Accordingly, it has been suggested that the patients with KD having lower PNI levels may be a potential candidate for an aggressive treatment strategy. Nevertheless, further studies should be warranted to verify the findings.

However, it is necessary to recognize limitations in the present study. First, the results have the potential to be biased in terms of the population choice. Second, although a multivariable analysis was used, the possibility of residual unmeasured covariables that may influence the outcomes cannot be excluded. For example, genetic, ethnic and regional factors, and IVIG metabolic factors have not been taken into account. Finally, the data sample used in the present study remains relatively small and only located in western China. Thus, these limitations can potentially limit the accuracy of the results in the present study. Therefore, the ability and accuracy of the PNI in predicting IVIG resistance should be further validated by performing additional prospective, multicenter studies.

\section{Conclusion}

In summary, to the best of our knowledge, the findings of the present study demonstrated for the first time that pretreatment PNI can be a valid predictive index for patients with IVIG resistance. Considering the convenience of this measure, PNI has great potential as a surrogate for further research and clinical application.

\section{Disclosures}

The authors have no potential conflicts of interest to declare with respect to authorship and/or publication of this article.

\section{Acknowledgements}

We acknowledge for their contribution Ju Zeng (The First People's Hospital of Yibin, SiChuan, China), Zhengxia Han (The Second People's Hospital of Yibin, SiChuan, China), Guoguang Jian (Luzhou people's Hospital, SiChuan, China) and Zhiling Ran (Southwest Medical University Affiliated Hospital of traditional Chinese Medicine, SiChuan, China).

\section{Author contributions}

Dr. Gang Li and Xiumei Xu conceived of and designed this study, designed the data collection instrument, joined the consensus conference, drafted the initial manuscript, and reviewed and revised the manuscript, approved the final manuscript as submitted and agree to be accountable for all aspects of the work. Ms. Yongying Gou, Ms. Rumeng Zeng, Ms. Yan Duan and Pengyuan Chen participated in the data collection. Dr. Bin Liu participated in the consensus conference, critically reviewed the manuscript for important intellectual content, approved the final manuscript as submitted and agreed to be accountable for all aspects of the work. Mr. Dong Liu, Ting Wan, Peng Jia and Pengyuan Chen analyzed the data and drafted the initial manuscript. All authors reviewed the manuscript.

\section{Competing interests}

The authors declare no competing interests.

\section{References}

[1] Burns JC, Capparelli EV, Brown JA, Newburger JW, Glode MP. Intravenous gamma-globulin treatment and retreatment in Kawasaki disease. US/Canadian Kawasaki Syndrome Study Group. Pediatr Infect Dis J. 1998. 17(12): 1144-8. 
[2] Wallace CA, French JW, Kahn SJ, Sherry DD. Initial intravenous gammaglobulin treatment failure in Kawasaki disease. Pediatrics. 2000. 105(6): E78.

[3] Wu S, Liao Y, Sun Y, et al. Prediction of intravenous immunoglobulin resistance in Kawasaki disease in children. World J Pediatr. 2020 .

[4] Chen Y, Hua Y, Zhang C, et al. Neutrophil-to-Lymphocyte Ratio Predicts Intravenous ImmunoglobulinResistance in Infants Under 12-Months Old With Kawasaki Disease. Front Pediatr. 2019. 7: 81.

[5] Wu G, Yue P, Ma F, Zhang Y, Zheng X, Li Y. Neutrophil-to-lymphocyte ratio as a biomarker for predicting the intravenous immunoglobulin-resistant Kawasaki disease. Medicine (Baltimore). 2020. 99(6): e18535.

[6] Ha KS, Lee J, Jang GY, et al. Value of neutrophil-lymphocyte ratio in predicting outcomes in Kawasaki disease. Am J Cardiol. 2015. 116(2): 301-6.

[7] Tan XH, Zhang XW, Wang XY, et al. A new model for predicting intravenous immunoglobin-resistant Kawasaki disease in Chongqing: a retrospective study on 5277 patients. Sci Rep. 2019. 9(1): 1722.

[8] Qi F, Zhou X, Wang Y, et al. Pre-treatment prognostic nutritional index may serve as a potential biomarker in urinary cancers: a systematic review and meta-analysis. Cancer Cell Int. 2018. 18: 207.

[9] Lu Z, Yan W, Liang J, et al. Nomogram Based on Systemic Immune-Inflammation Index to Predict Survival of Tongue Cancer Patients Who Underwent Cervical Dissection. Front Oncol. 2020. 10: 341.

[10] Huang X, Hu H, Zhang W, Shao Z. Prognostic value of prognostic nutritional index and systemic immune-inflammation index in patients with osteosarcoma. J Cell Physiol. 2019. 234(10): 18408-18414.

[11] Wang X, Wang Y. The prognostic nutritional index is prognostic factor of gynecological cancer: A systematic review and meta-analysis. Int J Surg. 2019. 67: 79-86.

[12] Hu Y, Shen J, Liu R, et al. Prognostic value of pretreatment prognostic nutritional index in non-small cell lung cancer: A systematic review and meta-analysis. Int J Biol Markers. 2018. 33(4): 372-378.

[13] Hayıroğlu MI, Keskin M, Keskin T, et al. A Novel Independent Survival Predictor in Pulmonary Embolism: Prognostic Nutritional Index. Clin Appl Thromb Hemost. 2018. 24(4): 633-639.

[14] Keskin M, Hayıroğlu MI, Keskin T, et al. A novel and useful predictive indicator of prognosis in STsegment elevation myocardial infarction, the prognostic nutritional index. Nutr Metab Cardiovasc Dis. 2017. 27(5): 438-446.

[15] Chen QJ, Qu HJ, Li DZ, et al. Prognostic nutritional index predicts clinical outcome in patients with acute ST-segment elevation myocardial infarction undergoing primary percutaneous coronary intervention. Sci Rep. 2017. 7(1): 3285 .

[16] Chen XL, Wei XB, Huang JL, et al. The prognostic nutritional index might predict clinical outcomes in patients with idiopathic dilated cardiomyopathy. Nutr Metab Cardiovasc Dis. 2020. 30(3): 393-399.

[17] Shirakabe A, Hata N, Kobayashi N, et al. The prognostic impact of malnutrition in patients with severely decompensated acute heart failure, as assessed using the Prognostic Nutritional Index (PNI) and Controlling Nutritional Status (CONUT) score. Heart Vessels. 2018. 33(2): 134-144.

[18] Tai IH, Wu PL, Guo MM, Lee J, Chu CH, Hsieh KS, Kuo HC. Prognostic nutrition index as a predictor of coronary artery aneurysm in Kawasaki Disease. BMC Pediatr. 2020. 20(1): 203.

[19] Newburger JW, Takahashi M, Gerber MA, Gewitz MH, Tani LY, Burns JC, Shulman ST, Bolger AF, Ferrieri P, Baltimore RS, Wilson WR, Baddour LM, Levison ME, Pallasch TJ, Falace DA, Taubert KA, Committee on Rheumatic Fever, Endocarditis, and Kawasaki Disease CoCDitY, Association AH. Diagnosis, treatment, and long-term management of Kawasaki disease: a statement for health professionals from the 
Committee on Rheumatic Fever, Endocarditis, and Kawasaki Disease, Council on Cardiovascular Disease in the Young, American Heart Association. Pediatrics. 2004. 114(6): 1708-1733.

[20] McCrindle BW, Rowley AH, Newburger JW, Burns JC, Bolger AF, Gewitz M, Baker AL, Jackson MA, Takahashi M, Shah PB, Kobayashi T, Wu MH, Saji TT, Pahl E, American Heart Association Rheumatic Fever, Endocarditis, Young aKDCotCoCDit, Nursing CoCaS, Anesthesia CoCSa, Prevention aCoEa. Diagnosis, Treatment, and Long-Term Management of Kawasaki Disease: A Scientific Statement for Health Professionals From the American Heart Association. Circulation. 2017. 135(17): e927-927e999.

[21] Fukunishi M, Kikkawa M, Hamana K, et al. Prediction of non-responsiveness to intravenous high-dose gamma-globulin therapy in patients with Kawasaki disease at onset. J Pediatr. 2000. 137(2): 172-6.

[22] Sleeper LA, Minich LL, McCrindle BM, et al. Evaluation of Kawasaki disease risk-scoring systems for intravenous immunoglobulin resistance. J Pediatr. 2011. 158(5): 831-835.e3.

[23] Cheng YL, Sung SH, Cheng HM, et al. Prognostic Nutritional Index and the Risk of Mortality in Patients With Acute Heart Failure. J Am Heart Assoc. 2017. 6(6).

[24] Min JY, Woo A, Chae MS, et al. Predictive Impact of Modified-Prognostic Nutritional Index for Acute Kidney Injury within 1-week after Living Donor Liver Transplantation. Int J Med Sci. 2020. 17(1): 82-88.

[25] Correa-Rodríguez M, Pocovi-Gerardino G, Callejas-Rubio JL, et al. The Prognostic Nutritional Index and Nutritional Risk Index Are Associated with Disease Activity in Patients with Systemic Lupus Erythematosus. Nutrients. 2019. 11(3).

[26] Ahn SS, Jung SM, Song JJ, Park YB, Lee SW. Prognostic nutritional index is correlated with disease activity in patients with systemic lupus erythematosus. Lupus. 2018. 27(10): 1697-1705.

[27] Yang S, Song R, Zhang J, Li X, Li C. Predictive tool for intravenous immunoglobulin resistance of Kawasaki disease in Beijing. Arch Dis Child. 2019. 104(3): 262-267.

[28] Kobayashi T, Inoue Y, Takeuchi K, et al. Prediction of intravenous immunoglobulin unresponsiveness in patients with Kawasaki disease. Circulation. 2006. 113(22): 2606-12.

[29] Do YS, Kim KW, Chun JK, Cha BH, Namgoong MK, Lee HY. Predicting factors for refractory kawasaki disease. Korean Circ J. 2010. 40(5): 239-42.

[30] Kil HR. Kawasaki disease and hyponatremia. Korean Circ J. 2010. 40(10): 489-90.

[31] Tsuji S. Significance of hyponatremia in Kawasaki disease. Pediatr Int. 2020. 62(3): 307.

[32] Kuo HC, Liang CD, Wang CL, Yu HR, Hwang KP, Yang KD. Serum albumin level predicts initial intravenous immunoglobulin treatment failure in Kawasaki disease. Acta Paediatr. 2010. 99(10): 1578-83.

[33] Colotta F, Allavena P, Sica A, Garlanda C, Mantovani A. Cancer-related inflammation, the seventh hallmark of cancer: links to genetic instability. Carcinogenesis. 2009. 30(7): 1073-81.

[34] Grivennikov SI, Greten FR, Karin M. Immunity, inflammation, and cancer. Cell. 2010. 140(6): 883-99.

[35] Zahorec R. Ratio of neutrophil to lymphocyte counts-rapid and simple parameter of systemic inflammation and stress in critically ill. Bratisl Lek Listy. 2001. 102(1): 5-14.

[36] Ray-Coquard I, Cropet C, Van Glabbeke M, et al. Lymphopenia as a prognostic factor for overall survival in advanced carcinomas, sarcomas, and lymphomas. Cancer Res. 2009. 69(13): 5383-91.

[37] Liu X, Zhou K, Hua Y, et al. Prospective Evaluation of Neutrophil-to-lymphocyte Ratio and Plateletto-lymphocyte Ratio for Intravenous Immunoglobulin Resistance in a Large Cohort of Kawasaki Disease Patients. Pediatr Infect Dis J. 2020. 39(3): 229-231.

[38] Takeshita S, Kanai T, Kawamura Y, Yoshida Y, Nonoyama S. A comparison of the predictive validity of the combination of the neutrophil-to-lymphocyte ratio and platelet-to-lymphocyte ratio and other risk 
scoring systems for intravenous immunoglobulin (ivig)-resistance in Kawasaki disease. PLoS One. 2017. 12(5): $\mathrm{e} 0176957$.

[39] Wu S, Long Y, Chen S, et al. A New Scoring System for Prediction of Intravenous Immunoglobulin Resistance of Kawasaki Disease in Infants Under 1-Year Old. Front Pediatr. 2019. 7: 514.

[40] Wakita M, Fukatsu A, Amagai T. Nutrition assessment as a predictor of clinical outcomes for infants with cardiac surgery: using the prognostic nutritional index. Nutr Clin Pract. 2011. 26(2): 192-8.

[41] Mirili C, Yılmaz A, Demirkan S, Bilici M, Basol Tekin S. Clinical significance of prognostic nutritional index (PNI) in malignant melanoma. Int J Clin Oncol. 2019. 24(10): 1301-1310.

[42] Sun Y, Huang Z, Chi P. An inflammation index-based prediction of treatment response to neoadjuvant chemoradiotherapy for rectal mucinous adenocarcinoma. Int J Clin Oncol. 2020 .

\section{Figure legends}

Figure 1. Receiver operating characteristic curves for pretreatment PNI, NLR and PLR based on IVIG resistance.

Figure 2. Association between PNI, NLR and PLR. (A) NLR and (B) PLR.

Table 1 Baseline patient characteristics

Characteristics

Age (months)

Median (Range)

Gender

Male

Female

White blood cell $\left(10^{9} / \mathrm{L}\right)$

Median (Range)

Hemoglobin (g/L)

Median (Range)

Platelet $\left(10^{9} / \mathrm{L}\right)$

$300\left(10^{9} / \mathrm{L}\right)$, median (Range)

$<300\left(10^{9} / \mathrm{L}\right)$, median (Range)

CRP (mg/L)

Median (Range)

$\operatorname{ESR}(\mathrm{mm} / \mathrm{h})$

Median (Range)

Serum sodium $(\mathrm{mmol} / \mathrm{L})$

Median (Range)

Total bilirubin (umol/L)

Median (Range)

IVIG resistance $(\mathrm{n}, \%)$

CRP: C-reactive protein; ESR:Erythrocyte sedimentation rate ; IVIG: intravenous immunoglobulin;
Table 1 Baseline pati

Number $(\%) /$ media

$19(11-38)$

$718(57.1 \%)$

$539(42.9 \%)$

$15.4(12.3-19.8)$

$106(97-119)$

?ं?

$378(254-463)$

$157(101-213)$

$83.4(48.7-119)$

$73(49.7-103.5)$

$135.7(133.84-137.19)$

$10.18(5.9-14.8)$

$187(14.9 \%)$

CRP: C-reactive prot

Table 2 Baseline patient characteristics based on PNI, NLR and PLR.

Characteristics

Age

12 months

$<12$ months

Gender 
Table 2 Baseline patient characteristics based on PNI, NLR and PLR.

Male

Female

Total bilirubin[?]9.7umol/L

No

Yes

Platelet $<300 \times 10^{9} / \mathrm{L}$

No

Yes

$\mathrm{CRP}[?] 78 \mathrm{mg} / \mathrm{L}$

No

Yes

Serum sodium $<135 \mathrm{mmol} / \mathrm{L}$

No

Yes

IVIG resistance

No

Yes

CRP: C-reactive protein; IVIG: intravenous immunoglobulin; PNI: prognostic nutritional index; NLR: neutrophil to lympho

\begin{tabular}{|c|c|c|c|c|c|c|c|}
\hline Table 3 & Table 3 & Table 3 & Table 3 & Table 3 & Table 3 & Table 3 & Table 3 \\
\hline $\begin{array}{l}\text { Univariate } \\
\text { and multi- } \\
\text { variate } \\
\text { analysis of } \\
\text { IVIG } \\
\text { resistant } \\
\text { KD. }\end{array}$ & $\begin{array}{l}\text { Univariate } \\
\text { and multi- } \\
\text { variate } \\
\text { analysis of } \\
\text { IVIG } \\
\text { resistant } \\
\text { KD. }\end{array}$ & $\begin{array}{l}\text { Univariate } \\
\text { and multi- } \\
\text { variate } \\
\text { analysis of } \\
\text { IVIG } \\
\text { resistant } \\
\text { KD. }\end{array}$ & $\begin{array}{l}\text { Univariate } \\
\text { and multi- } \\
\text { variate } \\
\text { analysis of } \\
\text { IVIG } \\
\text { resistant } \\
\text { KD. }\end{array}$ & $\begin{array}{l}\text { Univariate } \\
\text { and multi- } \\
\text { variate } \\
\text { analysis of } \\
\text { IVIG } \\
\text { resistant } \\
\text { KD. }\end{array}$ & $\begin{array}{l}\text { Univariate } \\
\text { and multi- } \\
\text { variate } \\
\text { analysis of } \\
\text { IVIG } \\
\text { resistant } \\
\text { KD. }\end{array}$ & $\begin{array}{l}\text { Univariate } \\
\text { and multi- } \\
\text { variate } \\
\text { analysis of } \\
\text { IVIG } \\
\text { resistant } \\
\text { KD. }\end{array}$ & $\begin{array}{l}\text { Univariate } \\
\text { and multi- } \\
\text { variate } \\
\text { analysis of } \\
\text { IVIG } \\
\text { resistant } \\
\text { KD. }\end{array}$ \\
\hline Variables & $\begin{array}{l}\text { Univariate } \\
\text { analysis } \\
\mathrm{HR}\end{array}$ & $\begin{array}{l}\text { Univariate } \\
\text { analysis } \\
95 \% \text { CI }\end{array}$ & $\begin{array}{l}\text { Univariate } \\
\text { analysis } \\
p \text { Value }\end{array}$ & & $\begin{array}{l}\text { Multivariate } \\
\text { analysis } \\
\mathrm{HR}\end{array}$ & $\begin{array}{l}\text { Multivariate } \\
\text { analysis } \\
95 \% \text { CI }\end{array}$ & $\begin{array}{l}\text { Multivariate } \\
\text { analysis } \\
p \text { Value }\end{array}$ \\
\hline Age & & & 0.031 & & & & 0.024 \\
\hline $\begin{array}{l}<12 \\
\text { months }\end{array}$ & Reference & & & & Reference & & \\
\hline $\begin{array}{l}{[?] 12} \\
\text { months }\end{array}$ & 0.939 & $\begin{array}{l}0.876- \\
0.989\end{array}$ & & & 0.914 & $\begin{array}{l}0.862- \\
0.978\end{array}$ & \\
\hline Gender & & & 0.45 & & & & \\
\hline Female & Reference & & & & Reference & & \\
\hline Male & 1.12 & $0.74-1.89$ & & & & & \\
\hline $\begin{array}{l}\text { Total } \\
\text { bilirubin } \\
{[?] 9.7} \\
\text { umol/L }\end{array}$ & & & 0.087 & & & & \\
\hline No & Reference & & & & Reference & & \\
\hline Yes & 1.33 & $0.8-2.21$ & & & & & \\
\hline $\begin{array}{l}\text { platelet < } \\
300 \times 10^{9} / \mathrm{L}\end{array}$ & & & 0.004 & & & & 0.079 \\
\hline No & Reference & & & & Reference & & \\
\hline Yes & 1.48 & $1.16-1.87$ & & & 1.22 & $0.89-1.51$ & \\
\hline
\end{tabular}




\begin{tabular}{|c|c|c|c|c|c|c|c|}
\hline $\begin{array}{l}\text { Table } 3 \\
\text { Univariate } \\
\text { and multi- } \\
\text { variate } \\
\text { analysis of } \\
\text { IVIG } \\
\text { resistant } \\
\text { KD. }\end{array}$ & $\begin{array}{l}\text { Table } 3 \\
\text { Univariate } \\
\text { and multi- } \\
\text { variate } \\
\text { analysis of } \\
\text { IVIG } \\
\text { resistant } \\
\text { KD. }\end{array}$ & $\begin{array}{l}\text { Table } 3 \\
\text { Univariate } \\
\text { and multi- } \\
\text { variate } \\
\text { analysis of } \\
\text { IVIG } \\
\text { resistant } \\
\text { KD. }\end{array}$ & $\begin{array}{l}\text { Table } 3 \\
\text { Univariate } \\
\text { and multi- } \\
\text { variate } \\
\text { analysis of } \\
\text { IVIG } \\
\text { resistant } \\
\text { KD. }\end{array}$ & $\begin{array}{l}\text { Table } 3 \\
\text { Univariate } \\
\text { and multi- } \\
\text { variate } \\
\text { analysis of } \\
\text { IVIG } \\
\text { resistant } \\
\text { KD. }\end{array}$ & $\begin{array}{l}\text { Table } 3 \\
\text { Univariate } \\
\text { and multi- } \\
\text { variate } \\
\text { analysis of } \\
\text { IVIG } \\
\text { resistant } \\
\text { KD. }\end{array}$ & $\begin{array}{l}\text { Table } 3 \\
\text { Univariate } \\
\text { and multi- } \\
\text { variate } \\
\text { analysis of } \\
\text { IVIG } \\
\text { resistant } \\
\text { KD. }\end{array}$ & $\begin{array}{l}\text { Table } 3 \\
\text { Univariate } \\
\text { and multi- } \\
\text { variate } \\
\text { analysis of } \\
\text { IVIG } \\
\text { resistant } \\
\text { KD. }\end{array}$ \\
\hline$\overline{\mathrm{CRP}[?] 78 \mathrm{mg} / \mathrm{I}}$ & & & $<0.001$ & & & & 0.006 \\
\hline No & Reference & & & & Reference & & \\
\hline Yes & 2.85 & $1.39-4.92$ & & & 1.17 & $1.07-1.25$ & \\
\hline $\begin{array}{l}\text { Serum } \\
\text { sodium } \\
<135 \\
\text { mmol/L } \\
\text { (n) }\end{array}$ & & & 0.028 & & & & 0.007 \\
\hline No & Reference & & & & Reference & & \\
\hline Yes & 1.5 & $1.166-1.95$ & & & 1.006 & $\begin{array}{l}1.001- \\
1.012\end{array}$ & \\
\hline PNI & & & $<0.001$ & & & & 0.001 \\
\hline $\begin{array}{l}<49.5 \\
{[?] 49.5}\end{array}$ & $\begin{array}{l}\text { Reference } \\
0.866\end{array}$ & $\begin{array}{l}0.831- \\
0.919\end{array}$ & & & $\begin{array}{l}\text { Reference } \\
0.852\end{array}$ & $\begin{array}{l}0.812- \\
0.898\end{array}$ & \\
\hline NLR & & & 0.003 & & & & 0.008 \\
\hline $\begin{array}{l}<3.58 \\
{[?] 3.58}\end{array}$ & $\begin{array}{l}\text { Reference } \\
1.369\end{array}$ & $1.126-1.58$ & & & $\begin{array}{l}\text { Reference } \\
1.33\end{array}$ & $\begin{array}{l}1.116- \\
1.532\end{array}$ & \\
\hline PLR & & & 0.009 & & & & 0.026 \\
\hline $\begin{array}{l}<164 \\
{[?] 164}\end{array}$ & $\begin{array}{l}\text { Reference } \\
1.74\end{array}$ & $1.08-2.85$ & & & $\begin{array}{l}\text { Reference } \\
1.3\end{array}$ & $\begin{array}{l}1.002- \\
1.684\end{array}$ & \\
\hline
\end{tabular}




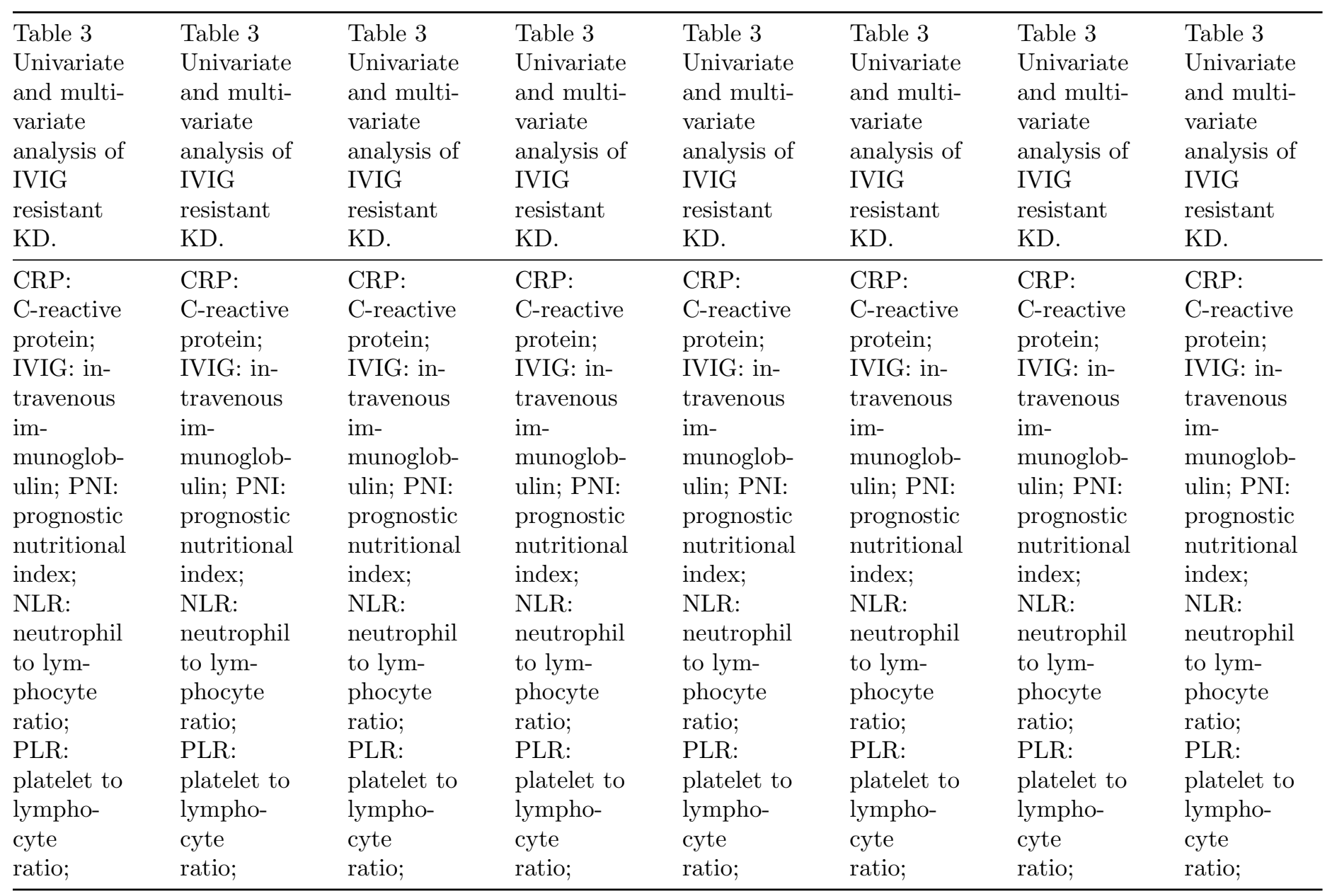

Figure 1 


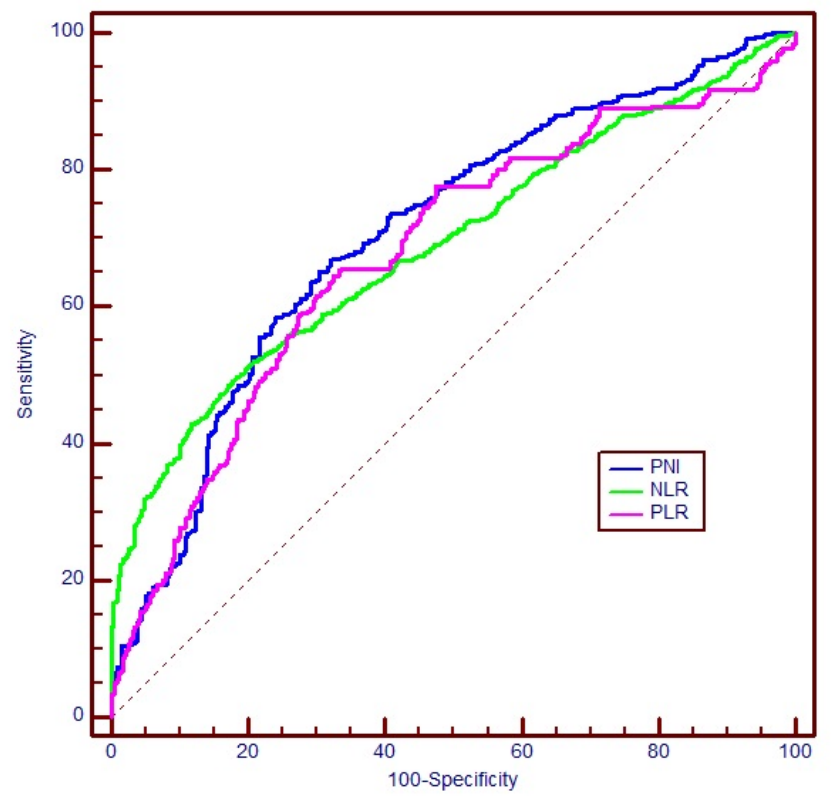

Figure 2
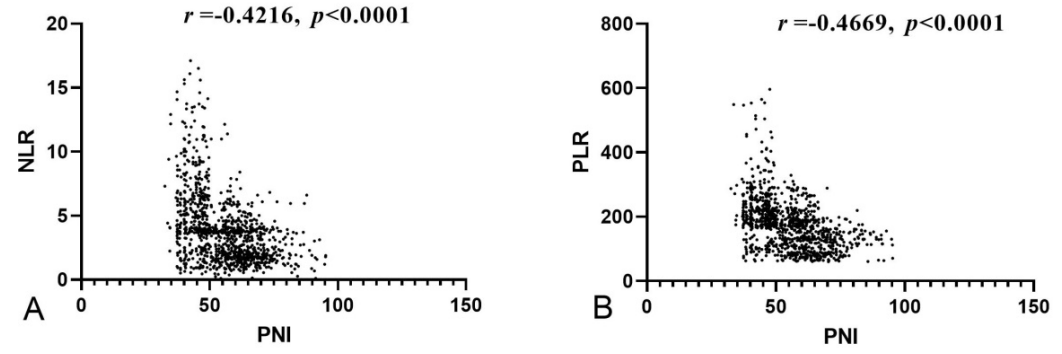